\title{
Transesophageal endoscopic ultrasound-guided fine needle aspiration for the diagnosis of vertebral infectious spondylodiscitis: a novel approach
}

\section{(웅 $\odot$}

\author{
Authors \\ Sofía Ibarra², Sonia Blanco ${ }^{1}$ \\ Institutions \\ 1 Gastroenterology Department, Hospital Universitario \\ Basurto, Bilbao, Spain \\ 2 Infectious Diseases Department, Hospital Universitario \\ Basurto, Bilbao, Spain
}

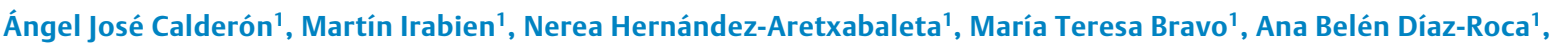

submitted 4.12.2019

accepted after revision 18.5 .2020

\author{
Bibliography \\ DOI https://doi.org/10.1055/a-1196-1421 | \\ Endoscopy International Open 2020; 08: E1031-E1033 \\ (c) Georg Thieme Verlag KG Stuttgart · New York \\ eISSN 2196-9736 \\ Corresponding author \\ Dr. Martín Irabien, Servicio Aparato Digestivo, Hospital \\ Universitario Basurto, Avenida Montevideo 18, 48013 \\ Bilbao, Spain \\ Fax: +1-0034944006180 \\ martin.irabienortiz@osakidetza.eus
}

Endoscopic ultrasound-guided fine-needle aspiration (EUSFNA) is a technique that enables study of human cells obtained through aspiration in different locations near the gastrointestinal tract. Access routes described in the literature to obtain samples of cervical and thoracic regions are open biopsy and computed tomography (CT)-guided biopsy. These techniques have some disadvantages as invasiveness and risk of complications in the case of open biopsy, as well as radiation emitted to the patient and not being a real time technique in the case of CT-guided biopsy.

We describe use of EUS-FNA as a novel approach able to target the cervical and thoracic regions in real time. We present our experience in the field of spondylodiscitis (cervical and thoracic), with the main objective of establishing debate on the possibilities of endoscopic ultrasound in this very complex anatomical area.

\section{Introduction}

The diagnosis of spondylodiscitis is established based on positive culture [1], either positive blood cultures in the absence of another possible focus ( $40 \%-80 \%$ positivity) or from biopsy of the involved vertebra(e), disc space and/or paravertebral abscess.

In general, biopsy is warranted to confirm clinical and/or radiographic suspicion of spondylodiscitis and to establish a microbiologic and histologic diagnosis. Biopsy material is usually obtained by an open procedure or by computed tomography (CT)-guided biopsy [2-5].

We report the cases of three patients admitted to our hospital in 2011, 2013, and 2017 with clinical and/or radiological suspicion of spondylodiscitis of thoracic and cervical vertebrae. We performed endoscopic ultrasound-guided fine-needle aspiration (EUS-FNA) to obtain samples of the involved vertebrae for microbiologic diagnosis.

\section{Procedure}

The technique was performed under endoscopist-guided deep sedation with propofol. There is a vast experience of sedation guided by endoscopists, in conditions of safety and efficacy, and it is backed up by the Spanish society of digestive endoscopy [6].

For the procedure, we used the Pentax-Hitachi sectoral echoendoscope. The distorted area of the woven bone was located by looking for the loss of posterior acustic shade from de cortical shell ( $\triangleright$ Fig. 1). It was punctured with a 22G Cook cytological aspiration needle to obtain enough material for diagnosis [7]. A total of two needle passes were performed for each procedure until we obtained enough sample.

The samples were sent to the microbiology and histopathology laboratories in a standardized manner.

For microbiology, samples were sent fresh (in a normal saline bottle) and aerobic, anaerobic, mycobacterial, and fungal 


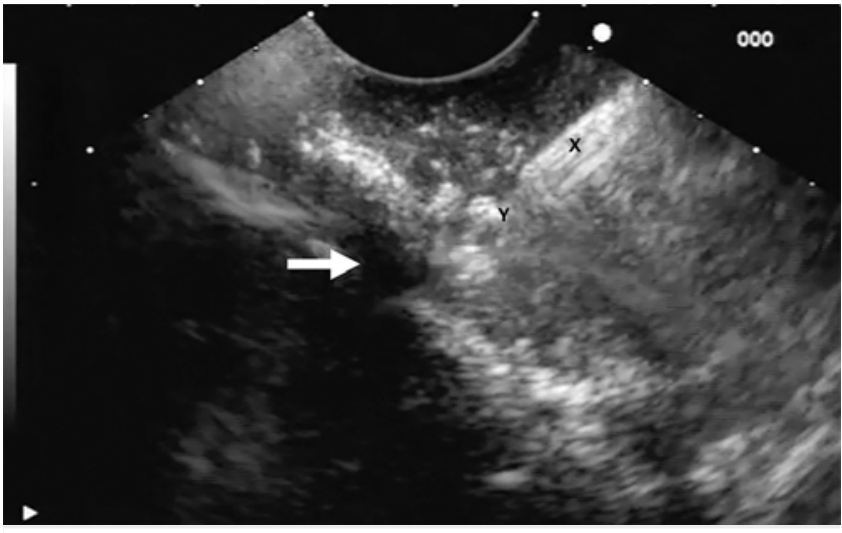

Fig. 1 Procedure. $x$, echoendoscope; $y$, needle; white arrow, distorted area with loss of posterior acoustic shade.

cultures were performed. Culture was carried out in basic (blood agar, chocolate agar and Brucella) and specific plates (Mc Conkey/Hektoen for gram negative and Columbia CNA for gram positive). In addition, an enriched medium was used anticipating that microbiological load was very low.

For the pathology laboratory, the stylet was first introduced and then the needle was flushed with a few milliliters of normal saline. A drop from the needle was expelled onto a glass slide and a single spread slide was made from each pass. No intraprocedure inmediate cytological assessment was performed, although it could be taken into account in the future.

\section{Results}

By using EUS-FNA, bacterial growth was obtained in $100 \%$ of cases.

In the first case, a 50-year-old man with suspected T5-spondylodiscitis and negative repeated blood cultures, microbiological study of the vertebra was positive for Staphylococcus aureus. In the second case, a 60-year-old woman with clinical suspicion of C5/C6-spondylodiscitis and negative repeated blood cultures, Streptococcus mitis was isolated. In the third and last case, a 65-year-old male with suspected C5-lesion who also had negative repeated blood cultures, two microorganisms grew: S. constellatus and S. mitis ( $>$ Table 1 ).

Cytological analysis of samples sent to the laboratory was negative for malignancy.
There were no complications associated with puncture of the esophageal wall with $22 \mathrm{G}$ needles or related to the introduction of the endoscope or deep sedation.

The results from the microbiological analysis helped us choose targeted antibiotic therapy. The clinical evolution of the three cases with different antibiotic regimens was good, allowing hospital discharge and subsequent follow-up through consultation.

\section{Discussion}

The pancreas and lymph nodes are still the most common organs targeted in EUS-FNA, and vertebral puncture, as we have done in our center, is not one of the well-established indications of echoendoscopy in clinical practice $[8,9]$. The main reason to perform an EUS-FNA in these cases of suspected spondylodiscitis was to confirm the diagnosis and to target antimicrobial therapy to the results of culture.

This is a relatively simple technique, with few associated complications. In the case of FNA, the main complication is bleeding, although it did not occur in our three cases. In reference to the number of passes, there is no scientific evidence in this regard, so we have followed the usual recommendations for that area and technique [10].

The microorganisms obtained in Cases 2 and 3 are wellknown commensals of the oral flora, so with the data obtained and in the absence of coincident positive blood cultures, we cannot be sure that they are not contaminants of the technique itself.

Considering the esophageal and spinal anatomy, this technique would be achievable in vertebrae between C4 and T5, always keeping in mind possible anatomical variants, so we think the approach could be useful in diagnosis of suspected vertebral lesions of non-infectious origin, especially cases in which CTguided biopsy cannot be performed.

We consider this technique a real-time approach with easier access than a CT-guided biopsy and with fewer described complications than open biopsy. But above all, the indication for performing this technique versus traditional transesophageal EUS-FNA was mainly the circumstances in our setting. For this reason, we want to emphasize that our objective is not to compare the technique we have presented with conventional techniques, but to share it as an alternative and open up scientific debate about its usefulness.

- Table 1 Results.

\begin{tabular}{|l|l|l|l|l|}
\hline & Sex & Age & Location & Microbiology \\
\hline Case 1 & Male & 50 & T5 & $\begin{array}{l}\text { Blood cultures: negatives } \\
\text { FNA culture: Staphylococcus aureus }\end{array}$ \\
\hline Case 2 & Female & 60 & C5-C6 & $\begin{array}{l}\text { Blood cultures: negatives } \\
\text { FNA culture: Streptococcus mitis }\end{array}$ \\
\hline Case 3 & Male & 65 & C5 & $\begin{array}{l}\text { Blood cultures: negatives } \\
\text { FNA culture: Streptococcus constellatus and Streptococcus mitis }\end{array}$ \\
\hline FNA, fine-needle aspiration. & & & \\
\hline
\end{tabular}


Therefore, given that this is a novel approach for which there are no precedents in the literature, further studies in a larger number of patients are required to assess our results and to provide stronger scientific evidence.

\section{Acknowledgements}

The authors thank the Gastroenterology Department of Hospital Universitario de Basurto for the training received.

\section{Competing interests}

The authors declare that they have no conflict of interest.

\section{References}

[1] Lew DP, Waldvogel FA. Osteomyelitis. Lancet 2004; 364: 369-379

[2] Chong BSW, Brereton C], Gordon A et al. Epidemiology, microbiological diagnosis, and clinical outcomes in pyogenic vertebral osteomyelitis: a 10-year retrospective cohort study. Open Forum Infect Dis 2018; 5: 1-6
[3] Marschall J, Bhavan KP, Olsen MA et al. The impact of prebiopsy antibiotics on pathogen recovery in hematogenous vertebral osteomyelitis. Clin Infect Dis 2011; 52: 867-872

[4] Hatzenbuehler J, Pulling TJ. Diagnosis and management of osteomyelitis. Am Fam Physician 2011; 84: 1027-1033

[5] Chang CY, Simeone FJ, Nelson SB et al. Is biopsying the paravertebral soft tissue as effective as biopsying the disk or vertebral endplate? 10-year retrospective review of CT-guided biopsy of diskitis-osteomyelitis AJR Am J Roentgenol 2015; 205: 123-129

[6] Igea F, Calderón Á, De-la-Peña J et al. Sedación en endoscopia digestiva. Guía de práctica clínica de la Sociedad Española de Endoscopia Digestiva. Rev Esp Enferm Dig 2014; 106: 195-211

[7] Carrara S, Auriemma F, Varadarajulu S. Advances in endoscopic ultrasound-guided tissue acquisition. Tech Gastrointest Endosc doi:10.1016/j.tgie.2017.10.006

[8] Hawes RH, Fockens P, Varadarajulu S. Endosonography. 3rd ed. Toronto: Saunders; 2015

[9] Dumonceau JM, Deprez PH, Jenssen C et al. Indications, results, and clinical impact of endoscopic ultrasound (EUS)-guided sampling in gastroenterology: European Society of Gastrointestinal Endoscopy (ESGE) Clinical Guideline - Updated January 2017. Endoscopy 2017; 49: $695-714$

[10] Liffman R, Courtman N. Fine needle aspiration of abdominal organs: a review of current recommendations for achieving a diagnostic sample. J Small Anim Pract 2017; 58: 599-609 